\title{
Plasma Concentrations of N-Terminal Pro-B-Type Natriuretic Peptide after caesarean section under spinal anaesthesia
}

\author{
F. Ferhi , A. Gharbi*, M. A. Bouslama, H. Khouadja , K. Tarmiz , K. Benjazia \\ Department of Anesthesiology and Critical Care Medicine. Farhat Hached University Teaching Hospital. Sousse. Tunisia \\ * Departement of cardiology Farhat Hached University Teaching Hospital. Sousse. Tunisia
}

\section{BACKGROUND AND GOAL OF STUDY:}

$\mathrm{N}$-terminal pro-B-type natriuretic peptide (NT-pro BNP) is a powerful predictor of cardiovascular outcome in many circumstances. Plasma concentrations of NT-pro BNP have been shown to reflect cardiac dysfunction and volume overload. During spinal anaesthesia for caesarean section, hemodynamic changes and the effects of various treatment regimens on NT-pro BNP are not studied.

The aim of this study was to assess serum levels of NT-pro BNP after caesarean delivery under spinal anaesthesia.

\section{MATERIALS AND METHODS:}

We conduct a prospective observational study at a tertiary level hospital over a period of 2 months. The study was approved by the hospital ethics committee. After receipt of informed consent, pregnant women proposed for caesarean sections under spinal anaesthesia were included. NT pro BNP level were measured at 2, 8 and 24 hours after delivery.

\section{RESULTS:}

One hundred and eighty-nine patients were enrolled in this study. The mean age was $32.4 \pm 5,7$ years. The mean gestational age was $36.48 \pm 1.55$ weeks of gestation. Fifty-six parturients $(29.6 \%)$ showed a significant increase in NT pro BNP level in postpartum period (Table. I). With these patients, a history of HTA ( 3 cases) and treated mitral valvular desease (2 cases) was noted.

The NTpro BNP elevation was associated with a Body Mass Index greater than $30 \mathrm{~kg} / \mathrm{m} 2$, ephedrine dose more than $30 \mathrm{mg}$ and oxytocin dose higher than $25 \mathrm{UI}$, preeclampsia and hemodynamic instability.

Echocardiography performed subsequently in positive patients showed hypertrophic cardiomyopathy in all preeclamptic patients and was normal in the remaining patients.

\begin{tabular}{ccc}
\multicolumn{2}{c}{ Table I: NT pro BNP levels in positive patients } \\
\hline & Number $(\%)$ & Mean \pm SD $(\mathrm{pg} / \mathrm{ml})$ \\
\hline NT pro BNP atH2 & $21(11,1)$ & $571,6 \pm 341,8$ \\
NT pro BNP at H8 & $26(13,7)$ & $1591,2 \pm 571,9$ \\
NT pro BNP at H24 & $26(13,7)$ & $1944,6 \pm 388$
\end{tabular}

\section{CONCLUSION:}

In our study, a significant increase in serum levels of NT pro BNP was observed in $29.6 \%$ of study patients. These results may reflect myocardium damage during cesarean delivery due to hemodynamic instability especially in presence of preeclampsia, which suggest that this peptide acts as a biomarker for high risk pregnancies.

Table II: Risk factor of NT pro BNP elevation

Univariate

$\begin{array}{cr}\text { NT pro BNP }(+) & \text { NT pro BNP } \\ n=56 & n=133\end{array}$

SBP $\geq 140$

20

\begin{abstract}
Oxytocin $>25 \mathrm{UI}$
\end{abstract}
Ephédrine $>30 \mathrm{mg}$

7

3

17

25

IMC $>\mathbf{3 0}$

28

3

3

66

0.009

6.01

0.011

86

$<0.001$

3.8

multivariate 